\title{
EFEITOS DA CANOAGEM ADAPTADA SOBRE O SISTEMA CARDIOPULMONAR DE PARAPLÉGICOS
}

\author{
EFFECTS OF ADAPTED CANOEING ON THE CARDIOPULMONARY SYSTEM OF PARAPLEGICS
}

EFECTOS DEL PIRAGÜISMO ADAPTADO SOBRE EL SISTEMA CARDIOPULMONAR DE PARAPLÉJICOS

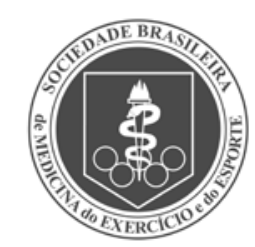

Artigo Original Original ARTICLE Artículo Original
Elizângela Márcia de Carvalho Abreu' (Fisioterapeuta)

Rani de Souza Alves²

(Engenheira Biomédica)

Leandro Orlandi Pereira' (Biólogo)

Fernanda Pupio Silva Lima'

(Fisioterapeuta)

Alderico Rodrigues de Paula Júnior ${ }^{3}$ (Engenheiro Elétrico)

Mário Oliveira Lima' (Fisioterapeuta)

1. Universidade do Vale do Paraíba (UNIVAP), São José dos Campos, SP, Brasil.

2. Instituto Nacional de Telecomunicações (INATEL), Santa Rita do Sapucaí, MG, Brasil. 3. Instituto Nacional de Pesquisas Espaciais (INPE), São José dos Campos, SP, Brasil.

\section{Correspondência:}

Laboratório de Engenharia de Reabilitação Sensório-Motora, UNIVAP. Av. Shishima Hifumi, 2911, Urbanova, São José dos Campos, SP, Brasil. 12244-000.

eliz_nasa@yahoo.com.br

\section{RESUMO}

Introdução: As doenças cardiovasculares e pulmonares estão entre as principais causas de morbidade e mortalidade de indivíduos com lesão medular (LM). O treinamento físico pode ser uma alternativa terapêutica para prevenir ou amenizar complicações cardiopulmonares nessa população. Objetivo: Avaliar os efeitos do treinamento com canoagem adaptada, constituído por exercícios combinados (aeróbicos e de força), sobre variabilidade da frequência cardíaca (VFC), função pulmonar e força muscular respiratória de pessoas paraplégicas em decorrência da LM. Métodos: Participaram do estudo seis paraplégicos, cinco homens e uma mulher, média de idade de 31,50 7,68 anos e índice de massa corporal médio de $24,00 \pm 1,13 \mathrm{~kg} / \mathrm{m}^{2}$, nível de lesão de T4 a T9, que realizaram treinamento com canoagem adaptada por três meses. Os indivíduos foram avaliados por VFC, espirometria e manovacuometria antes e depois do treinamento. Resultados: Após o treinamento houve aumento não significativo de RR (12,7\%), SDNN (24,3\%), rMSSD (50,0\%), pNN50 (478,6\%), LF (ms²) (53,3\%), HF $\left(\mathrm{ms}^{2}\right)$ (158,8\%), SD1 (50,6\%), SD2 (23,2\%) e SampEn (20,2\%). Os índices HF (u.n) e LF/HF tiveram redução não significativa de 5,7 e 7,0\%, respectivamente. Os maiores ganhos respiratórios foram para VVM $(9,7 \%), \mathrm{Pi}_{\text {máx }}(8,5 \%)$ e $\mathrm{Pe}_{\text {máx }}(11,0 \%)$, porém, não foram significativos. Conclusão: O protocolo proposto de três meses de canoagem adaptada não foi capaz de promover efeitos significativos sobre os parâmetros cardiopulmonares avaliados em indivíduos paraplégicos pós-LM, porém se observa uma tendência de melhora da maioria desses parâmetros. Nesse sentido, provavelmente, o estímulo de treinamento foi insuficiente.

Descritores: paraplegia; esportes para pessoas com deficiência; sistema nervoso autônomo; testes de função respiratória.

\section{ABSTRACT}

Introduction: Cardiovascular and pulmonary diseases are among the leading causes of morbidity and mortality in individuals with spinal cord injury (SCI). Physical training can be an alternative therapy to prevent or mitigate cardiopulmonary complications in this population. Objective: To evaluate the effects of training with adapted canoeing, consisting of combined exercise (aerobic and strength) on heart rate variability (HRV), pulmonary function and respiratory muscle strength in paraplegics due to SCI. Methods: The study included six paraplegics, five men and one woman, average age of $31.50 \pm 7.68$ years and mean body mass index of $24.00 \pm 1.13 \mathrm{~kg} / \mathrm{m}^{2}$, level of injury T4 to T9, who underwent training with adapted canoeing for three months. Subjects were evaluated by HRV, spirometry and manovacuometer before and after the training period. Results: After training there was no significant increase in RR (12.7\%), SDNN (24.3\%), rMSSD (50.0\%), pNN50 (478.6\%), LF (ms $)^{2}$ (53.3\%), HF (ms') (158.8\%) SD1 (50.6\%), SD2 (23.2\%) and SampEn (20.2\%). The HF indices (un) and LF/HF showed no significant decrease of 5.7 and $7.0 \%$, respectively. The highest breathing gains were for MVV (9.7\%), MIP (8.5\%) and MEP (11.0\%), being however not significant. Conclusion: The proposed protocol of three months of adapted canoeing failed to promote significant effects on cardiopulmonary parameters evaluated in paraplegic subjects after SCl, but we observe the trend of improvement in most of these parameters. In this sense, probably the training stimulus was insufficient.

Keywords: paraplegia; sports for persons with disabilities; autonomic nervous system; respiratory function tests.

\section{RESUMEN}

Introducción: Las enfermedades cardiovasculares y pulmonares se encuentran entre las principales causas de morbilidad y mortalidad en las personas con lesión de la médula espinal (LME). El entrenamiento físico puede ser una alternativa terapéutica para prevenir o mitigar las complicaciones cardiopulmonares en esta población. Objetivo: Evaluar los efectos del entrenamiento con piragüismo adaptado, que consiste en el ejercicio combinado (aeróbico y fuerza) en la variabilidad de la frecuencia cardiaca (VFC), l a función pulmonary la fuerza de los músculos respiratorios en los parapléjicos debido a LME. Métodos: El estudio incluyó seis parapléjicos, cinco hombres y una mujer, la edad promedio fue $31.50 \pm 7,68$ años y el indice de mas a corporal promedio fue $24,00 \pm 1,13 \mathrm{~kg} / \mathrm{m}^{2}$ y nivel de la lesión de T4 a T9, que se sometieran a entrenamiento de piragüismo adaptado con duración de tres meses. Los sujetos fueron evaluados por la VFC, la espirometría y el manovacuómetro antes y después del entrenamiento. Resultados: Después del entrenamiento no hubo un aumento significativo en RR (12,7\%), $\operatorname{SDNN}(24,3 \%), \operatorname{rMSSD}(50,0 \%), \operatorname{pNN} 50(478,6 \%)$, LF $\left(m s^{2}\right)(53,3 \%), \operatorname{HF}\left(\mathrm{ms}^{2}\right)(158,8 \%), \operatorname{SD} 1(50,6 \%), \mathrm{SD} 2(23,2 \%)$ y SampEn (20,2\%). Los índices de HF (u.n) y LF/HF tuvieron 
una reducción no significativa de 5,7\% y 7,0\%, respectivamente. Las mayores ganancias respiratorias fueran de MWV (9,7\%), $P I_{\text {máx }}(8,5 \%)$ y $P E_{\text {máx }}(11,0 \%)$, sin embargo, no fueron significativas. Conclusión: El protocolo propuesto de tres meses de piragüismo adaptado no fue capaz de promover efectos significativos en los parámetros cardiopulmonares evaluados en parapléjicos post-LME, pero se observó una tendencia al mejoramiento de la mayoría de estos parámetros. En este sentido, probablemente el estímulo de entrenamiento ha sido insuficiente.

Descriptores: paraplejí; deportes para personas con discapacidad; sistema nervioso autónomo; pruebas de función respiratoria.

\section{INTRODUÇÃO}

Estudos apontam perda da inervação simpática, com preservação parassimpática, via nervo vago, em indivíduos com lesão medular (LM) acima do segmento T61,2, o que resulta em alterações cardiovasculares como redução da variabilidade da frequência cardíaca (VFC) e da variabilidade da pressão arterial, redução do componente de baixa frequência (LF) da potência espectral da série temporal dos intervalos RR, atraso do barorreflexo ${ }^{3}$, bradicardia, hipotensão arterial, disreflexia autonômica'; e em alterações broncopulmonares como: hipersensibilidade e redução do calibre das vias aéreas ${ }^{4}$.

Embora a inervação simpática esteja preservada em pessoas com LM abaixo do segmento T6, podem ocorrer alterações do controle autonômico cardiovascular, como diminuição do componente de alta frequência (HF) em repouso ${ }^{3}$, menor complexidade da frequência cardíaca (FC) em repouso, durante exercício aeróbico ${ }^{5}$ e durante exercício estático ${ }^{6}$. Associado a alteração autonômica podem ocorrer vasoplegia, diminuição da ativação da bomba muscular e consequente diminuição do retorno venoso? ${ }^{7}$.

Além do comprometimento cardiovascular a LM pode prejudicar a função dos músculos intercostais e abdominais, acarretando em redução dos volumes pulmonares, dificuldade de expansão total da caixa torácica, tosse ineficaz e dificuldade de remoção de secreções ${ }^{4,8}$.

A inatividade física, muito comum entre indivíduos com LM, predispõe ao aparecimento de doenças cardiovasculares ${ }^{1,3,6}$ e pulmonares ${ }^{4,9}$, principais causas de morbidade e mortalidade nesta população. Por isso, o processo de reabilitação deve incluir estratégias para prevenir ou pelo menos amenizar tais doenças. Sheel et al. ${ }^{8}$ demonstraram fortes evidências que apoiam o uso do treinamento físico nesta população para induzir melhoras cardiopulmonares.

Um esporte que pode ser utilizado por pessoas com LM é a canoagem adaptada. Na canoagem adaptada pode-se ou não utilizar equipamentos que auxiliem o praticante a desenvolver o seu melhor rendimento com segurança ${ }^{10}$. Não há diferenças em relação à técnica da remada para pessoas sem $L M$, apenas a pessoa com LM pode não conseguir realizar a fase de tração da remada por deficiência de equilíbrio e rotação de tronco ${ }^{11}$. Estudos têm demonstrado os efeitos do treinamento com canoagem de alto rendimento sobre a capacidade aeróbia e anaeróbica em atletas sem $\mathrm{LM}^{12,13}$, porém, não há registro sobre seus efeitos em pessoas com LM.
Pouco conhecimento existe sobre o controle autonômico cardiovascular quando as vias neurais foram comprometidas, além disso, a influência do treinamento com canoagem adaptada sobre a modulação autonômica cardiovascular após LM ainda não foi estabelecida. Da mesma forma, a atuação do treinamento físico sobre a função pulmonar após LM precisa ser melhor investigada. Portanto, o objetivo do estudo foi avaliar a VFC, a função pulmonar e a força muscular respiratória de pessoas paraplégicas em decorrência da LM, antes e após um programa de treinamento utilizando a canoagem adaptada.

\section{MÉTODOS}

Trata-se de um estudo experimental, não controlado e longitudinal, aprovado pelo Comitê de Ética em Pesquisa em Seres Humanos do UNIVAP, sob o número 18353613.0.0000.5503 e registrado no Clinical Trials, sob o número NCT02177929. Todos os pacientes leram e assinaram o Termo de Consentimento Livre e Esclarecido.

Foram selecionados 15 indivíduos paraplégicos após LM, nove deles não se enquadraram nos critérios de inclusão estabelecidos nessa pesquisa, tais como, idade entre 18 e 48 anos, LM torácica completa ou incompleta, com tempo de lesão acima de oito meses, espasticidade de membros inferiores igual ou inferior a 2 (escala de Ashworth modificada), estar clinicamente estável, apresentar atestado médico de liberação para a prática de atividade física, não possuir deformidade, contratura grave, doenças respiratórias associadas, anormalidades cardiovasculares previamente diagnosticadas ou fatores de risco como hipertensão, diabetes e obesidade, uso de medicações cardiodepressoras e cardioestimuladoras, não possuir úlceras de decúbito infectadas ou sem cobertura adequada com curativo impermeável, micoses, processos inflamatórios e neoplasias.

Como fatores de exclusão foram considerados: hidrofobia, dor neuropática importante, infecção de urina grave, gravidez e assiduidade aos treinos menor que $80 \%$.

A amostra do estudo, caracterizada na Tabela 1, foi constituída por seis paraplégicos (cinco homens e uma mulher), todos sedentários.

\section{Procedimentos de avaliação}

Inicialmente, os indivíduos passaram por consulta com a equipe de enfermagem da UNIVAP para avaliação da integridade da pele

Tabela 1. Caracterização clínica e antropométrica da amostra.

\begin{tabular}{|c|c|c|c|c|c|c|c|c|}
\hline Indivíduos & Idade (anos) & Peso (kg) & Estatura (m) & IMC $\left(\mathrm{kg} / \mathrm{m}^{2}\right)$ & Tempo de LM (meses) & Nível de LM & AIS & Etiologia \\
\hline 1 & 29 & 70 & 1,70 & 24,22 & 8 & $\mathrm{~T} 4$ & A & FAF \\
\hline 2 & 45 & 65 & 1,67 & 23,30 & 21 & T9 & C & Ac. trânsito \\
\hline 3 & 26 & 70 & 1,75 & 22,85 & 27 & $\mathrm{~T} 7$ & D & Ac. trânsito \\
\hline 4 & 23 & 78 & 1,75 & 25,46 & 42 & T9 & A & Ac. trânsito \\
\hline 5 & 33 & 65 & 1,67 & 23,30 & 24 & $\mathrm{~T} 5$ & A & Ac. trânsito \\
\hline 6 & 33 & 88 & 1,86 & 25,43 & 156 & $\mathrm{~T} 4$ & A & Ac. trânsito \\
\hline Média土DP & $31,50 \pm 7,68$ & $72,66 \pm 8,89$ & $1,73 \pm 0,07$ & $24,00 \pm 1,13$ & $46,33 \pm 54,82$ & - & - & - \\
\hline
\end{tabular}

IMC: índice de massa corpórea; LM: lesão medular; DP: desvio padrão; FAF: ferimento por arma de fogo, Ac. trânsito: acidente de trânsito. AIS: American Spinal Injury Association Impairment Scale. 
(quanto a úlceras de pressão e micoses), e receberam orientações e cuidados necessários.

Os indivíduos foram avaliados antes e após o período de treinamento, considerando a VFC e os testes respiratórios (espirometria e manovacuometria). As coletas foram realizadas pela manhã, nos dias em que os indivíduos não realizaram os treinos.

\section{Variabilidade da frequência cardíaca}

Os intervalos entre as ondas $R$ do eletrocardiograma (RR) foram coletados por meio de um monitor cardíaco Polar RS800CX (Kempele, Finlândia). A coleta foi realizada durante 7 minutos, sendo estes precedidos de pelo menos dois minutos de repouso na posição sentada com os membros superiores apoiados nas coxas. A cinta do polar foi posicionada abaixo dos músculos peitorais.

Os dados adquiridos foram transferidos para um microcomputador equipado com o software Polar ProTrainer (Kempele, Finlândia). Os sinais foram filtrados manualmente ${ }^{2,14}$ para eliminar os artefatos, e analisados por métodos lineares (domínio do tempo e da frequência) e não lineares (plotagem de Poincaré e entropia amostral), utilizando o software Kubios HRV Analysis ${ }^{\circledR}$ (Kuopio, Finlândia).

Da análise temporal foram obtidas as variáveis: RR, o desvio padrão dos intervalos RR (SDNN, do inglês standard deviation of all NN Intervals), a raiz quadrada da média das diferenças sucessivas entre intervalos RR (rMSSD, do inglês square root of the mean of the sum of the squares of differences between adjacent NN intervals), a porcentagem das diferenças sucessivas entre os intervalos RR adjacentes maiores que 50 ms (pNN50, do inglês number of pairs of adjacent NN intervals differing by more than $50 \mathrm{~ms}$ ).

Para a análise espectral, a transformada rápida de Fourier foi utilizada para se obter: LF, do inglês Low Frequency $(0,04-0,15 \mathrm{~Hz}), \mathrm{HF}$, do inglês High Frequency $(0,15-0,4 \mathrm{~Hz})$ e a razão LF/HF, em unidades absolutas $\left(\mathrm{ms}^{2}\right.$ ) e unidades normalizadas (u.n).

Com a utilização da plotagem de Poincaré foram obtidos: o desvio padrão da variabilidade instantânea batimento a batimento (SD1), relacionado com a variabilidade de curto prazo da FC e é influenciado principalmente pela modulação parassimpática, e o desvio padrão em longo prazo dos intervalos RR contínuos (SD2) medida da variabilidade de longo prazo e reflete a ativação simpática ${ }^{15}$.

Além disso, foi utilizada a análise por entropia amostral (SampEn), que indica a complexidade ou irregularidade da série temporal $R R$, $O$ comprimento $(m)$ dos segmentos e o intervalo de tolerância $(r)$ foram definidos em 2 e 0,2, respectivamente ${ }^{14}$. As medidas da complexidade da FC pode representar um marcador geral simpatovagal, o seu aumento reflete melhora no balanço autonômico, e sua diminuição está relacionada ao envelhecimento e às doenças cardiovasculares ${ }^{14}$.

Merati et al. ${ }^{5}$ e Agiovlasitis et al. ${ }^{6}$ sugeriram que as alterações autonômicas associadas a LM podem não ser reveladas pelos índices HF e LF/HF, por outro lado a análise de entropia foi bastante sensível. Por isso, o presente estudo propôs analisar a VFC por métodos lineares e não lineares.

Para análise foram excluídos os sinais obtidos no primeiro e no último minuto de coleta.

\section{Testes respiratórios}

Inicialmente os indivíduos foram cuidadosamente orientados quanto aos procedimentos a serem adotados. As coletas foram realizadas com os indivíduos sentados em suas cadeiras de rodas. Para evitar o vazamento de ar, utilizou-se um clipe nasal durante as coletas. Os indivíduos foram orientados a atingir o esforço máximo e mantê-lo pelo tempo necessário em cada manobra.

\section{Espirometria}

Para realização da espirometria foi utilizado um espirômetro computadorizado, com sensor de turbina a fluxo (Microquark, da marca Cosmed ${ }^{\circledR}$, equipado com o software PFT suite, versão 9.1b, Roma, Itália), conectado a um bucal de plástico rígido $(6,5 \mathrm{~cm}$ de comprimento e 2,5 cm de diâmetro).

As seguintes manobras foram avaliadas: capacidade vital lenta, capacidade vital forçada (CVF) e ventilação voluntária máxima (VM) para avaliação da função pulmonar, de acordo com o Consenso Brasileiro de Espirometria ${ }^{16}$. Os parâmetros avaliados foram: CVF, pico de fluxo expiratório (PFE), volume expiratório forçado no primeiro segundo (VEF), índice de Tiffeneau (VEF $/$ /CVF\%), fluxo expiratório forçado médio (FEF 25-75\%) e VMM. Cada manobra foi repetida por três vezes ${ }^{16}$, e o maior valor foi escolhido para análise.

\section{Manovacuometria}

As pressões respiratórias máximas foram mensuradas por meio de um manovacuômetro analógico da marca Ger-Ar ${ }^{\circledR}$ (São Paulo, Brasil), escalonado de $-300 \mathrm{a}+300 \mathrm{cmH}_{2} \mathrm{O}$, conectado a uma traqueia $(13,5$ $\mathrm{cm}$ de comprimento e $2,5 \mathrm{~cm}$ de diâmetro), um bucal plástico rígido ( $6 \mathrm{~cm}$ de comprimento e $2 \mathrm{~cm}$ de diâmetro). O sistema continha um orifício de fuga de um milímetro em sua extremidade distal ${ }^{17}$.

A pressão inspiratória máxima $\left(\mathrm{PI}_{\max }\right)$ foi obtida por uma manobra de inspiração máxima executada a partir do volume residual e a manobra de pressão expiratória máxima $\left(P E_{\max }\right)$ por uma manobra de expiração máxima precedida de uma inspiração máxima, ao nível da capacidade pulmonar total ${ }^{17}$, com as bochechas pressionadas para que não houvesse retenção de ar no espaço entre os dentes e as bochechas ${ }^{18}$.

Cada manobra foi realizada no mínimo três vezes, com um intervalo de descanso de um a dois minutos entre as medições ${ }^{19}$. As pressões sustentadas por pelo menos dois segundos foram registradas. $O$ valor selecionado para análise foi o maior encontrado de três medidas com variação menor que $10 \%$ entre elas ${ }^{20}$.

\section{Protocolo de treinamento com Canoagem Adaptada}

Antes e após os treinos, os sinais vitais e o estado geral de saúde dos indivíduos foram avaliados. Além disso, os indivíduos foram orientados quanto ao esvaziamento da bexiga e uso de curativo impermeável para úlcera de pressão (indivíduos 2 e 5) antes de entrar no caiaque.

As duas primeiras aulas foram destinadas a preparação do indivíduo fora d'água quanto à técnica da remada, e na água quanto ao treino de queda do caiaque e retorno a superfície da água com segurança, além do treino de remada e domínio do caiaque, sempre com a supervisão de um profissional especializado.

Os indivíduos foram submetidos ao treinamento de canoagem adapatada por cerca de 60 minutos. A frequência de treinamento foi de duas vezes por semana, sempre nos mesmos dias da semana, com intervalo de pelo menos dois dias entre os treinos, esta frequência de treinamento é apoiada por Tawashy et al. ${ }^{21}$. A intensidade do exercicio variou de 45 a 65\% da FC de reserva, a percepção subjetiva de esforço, avaliada por meio da escala de 20 pontos de Borg ${ }^{21,22}$, variou de 9 a 14 pontos.

Os treinos foram realizados na piscina da clínica escola/UNIVAP (comprimento de $11 \mathrm{~m}$ e largura de $5 \mathrm{~m}$, profundidade de 1,40 m, temperatura da água $34^{\circ} \mathrm{C}$ ), sempre no período da manhã, por um período de treinamento de três meses. Foi utilizado um caiaque (Eko, modelo solar individual, São Paulo, Brasil), duas faixas de EVA (43 cm de comprimento e de $10 \mathrm{~cm}$ de largura) para apoiar os maléolos laterais, evitando assim atrito contra o caiaque e possível úlcera de pressão, um 
encosto (46 cm de comprimento e $35 \mathrm{~cm}$ de largura) para auxiliar no equilíbrio de tronco, utilizado apenas quando necessário, geralmente durante o primeiro mês apenas.

O protocolo de treinamento com canoagem adaptada, constituído por exercícios aeróbicos e de força, foi dividido em três fases e está descrito no Anexo 1.

Durante o período de treinamento os indivíduos foram instruídos a não iniciarem nenhuma outra atividade, seja ela de reabilitação ou lazer.

\section{Análise dos dados}

A normalidade dos dados foi verificada utilizando os testes de Lilliefors e de valores extremos. O teste $t$ de Student foi utilizado para realizar as comparações entre as médias dos parâmetros avaliados pré e pós-treinamento $(p<0,05)$. Para analisar os dados os softwares Excel2010 (Redmond, Washington, Estados Unidos) Bioestat 5.0 (Tefé, Amazonas, Brasil) e Origin (Northampton Massachusetts, Estados Unidos) foram utilizando. Os dados foram expressos em média e erro padrão para os parâmetros da VFC e em média e desvio padrão para os parâmetros de espirometria e manovacuometria e também em porcentagem.

\section{RESULTADOS}

Os resultados da análise da VFC, expressos nas Figuras 1, 2 e 3, mostraram que não houve diferenças estatisticamente significativas antes e após o treinamento, porém houve aumento do RR de 12,7\%, SDNN de 24,3\%, rMSSD de 50,0\%, pNN50 de 478,6\%, LF (ms ${ }^{2}$ ) de 53,3\%, HF $\left(\mathrm{ms}^{2}\right)$ de $158,8 \%$, SD1 de 50,6\%, SD2 de 23,2\% e SampEn de 20,2\%. Os índices HF (u.n) e LF/HF sofreram uma redução após o treinamento de 5,7 e 7,0\%, respectivamente.

Os resultados da análise espirométrica mostraram que não houve diferenças estatisticamente significativas antes e após o treinamento, porém houve aumento do PFE (L/S) de 11,1\%, e WM (\%) de 9,7\% (Figura 4). Para os parâmetros expressos em porcentagem do previsto houve um aumento após o treinamento de 5,9\% para CVF, de 5,2\% para VEF1, de 6,3\% para PFE e de 10,4\% para WM (Figura 5).

Os resultados da análise das pressões respiratórias máximas demonstraram que não houve diferenças estatisticamente significativas antes e após o treinamento, porém a $\mathrm{Pi}_{\max }$ inicial passou de $-126,67 \pm 30,60 \mathrm{cmH}_{2} \mathrm{O}$ e para $-137,50 \pm 46,01 \mathrm{cmH}_{2} \mathrm{O}$ e a $\mathrm{Pe}_{\max }$ passou de 113,33 $\pm 42,26 \mathrm{cmH}_{2} \mathrm{O}$ para 125,83 $\pm 57,65 \mathrm{cmH}_{2} \mathrm{O}$ após o treinamento (Figura 6).

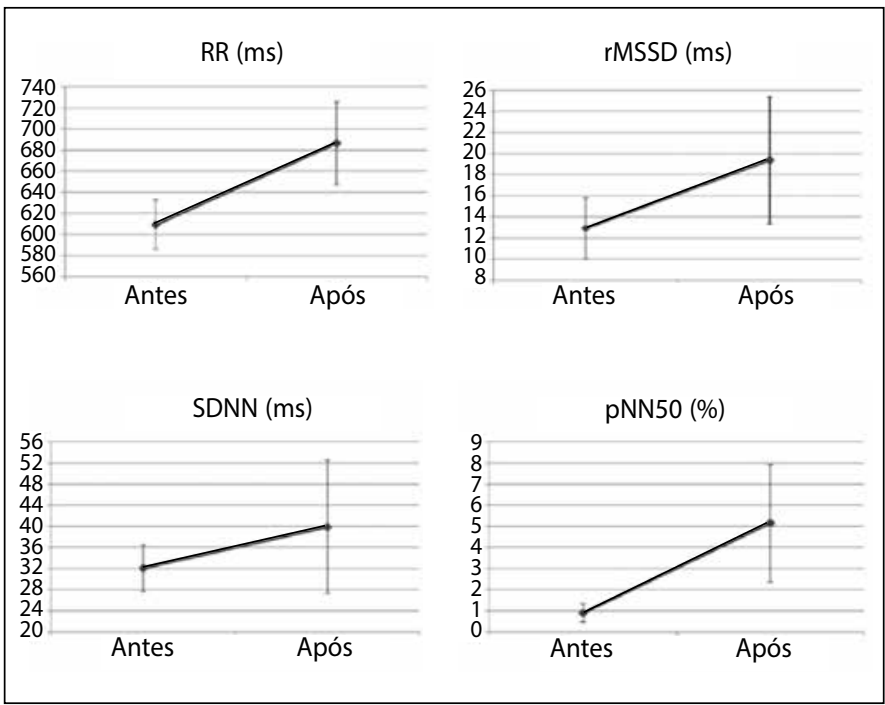

Figura 1. Valores em média e erro padrão dos parâmetros de variabilidade da frequência cardíaca analisados por métodos lineares no domínio do tempo antes e após o treinamento.

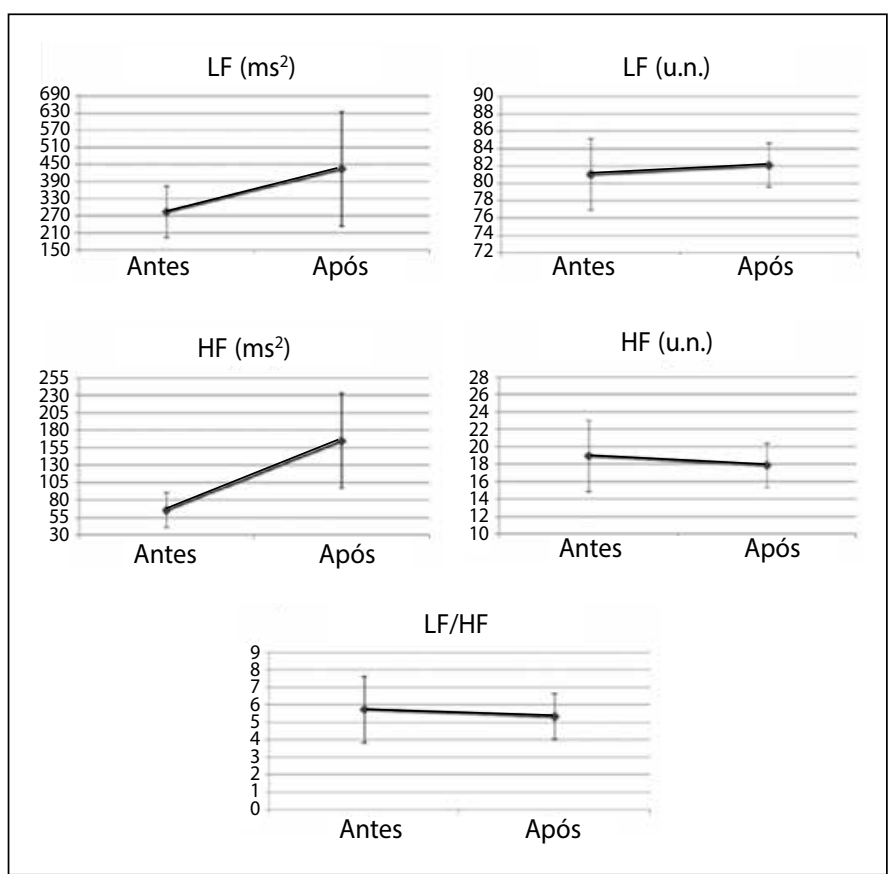

Figura 2. Valores em média e erro padrão dos parâmetros de variabilidade da frequência cardíaca analisados por métodos lineares no domínio da frequência antes e após o treinamento.

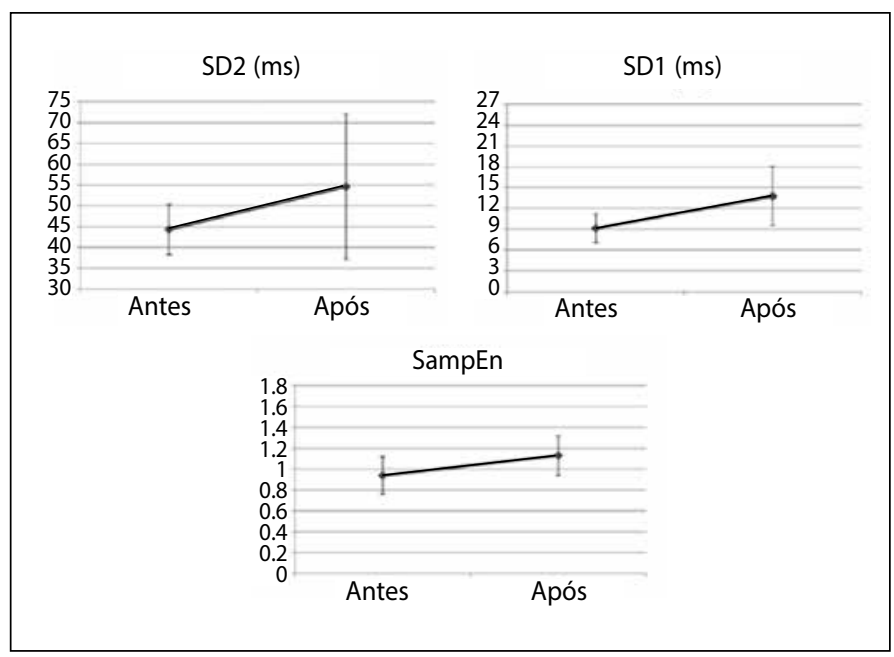

Figura 3. Valores em média e erro padrão dos parâmetros de variabilidade da frequência cardíaca analisados por métodos não lineares (plotagem de Poincaré e entropia amostral) antes e após o treinamento.

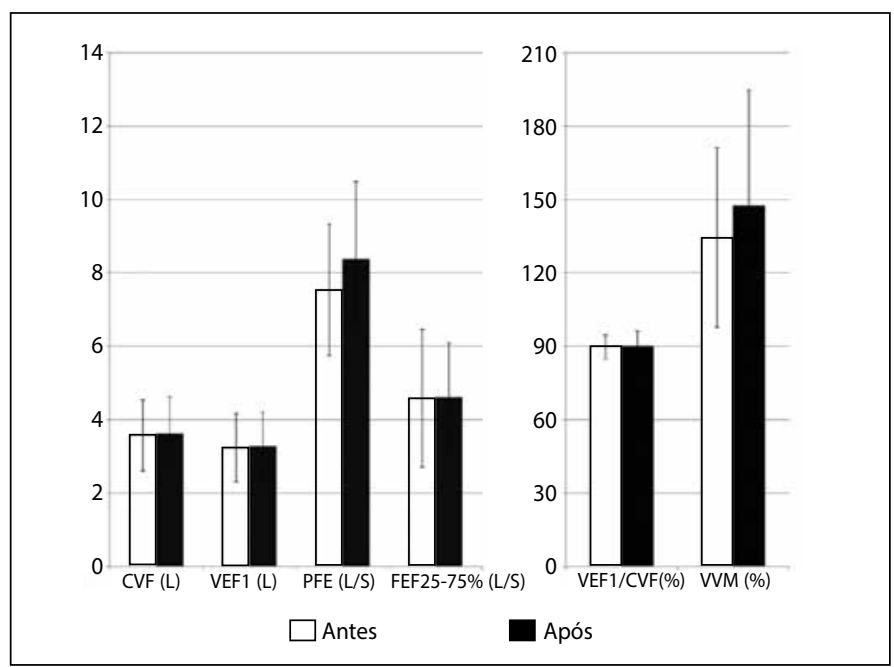

Figura 4. Valores espirométricos em média e desvio padrão antes e após o treinamento, expressos em litros $(\mathrm{L})$, litros por segundo (L/s) ou em porcentagem (\%). 


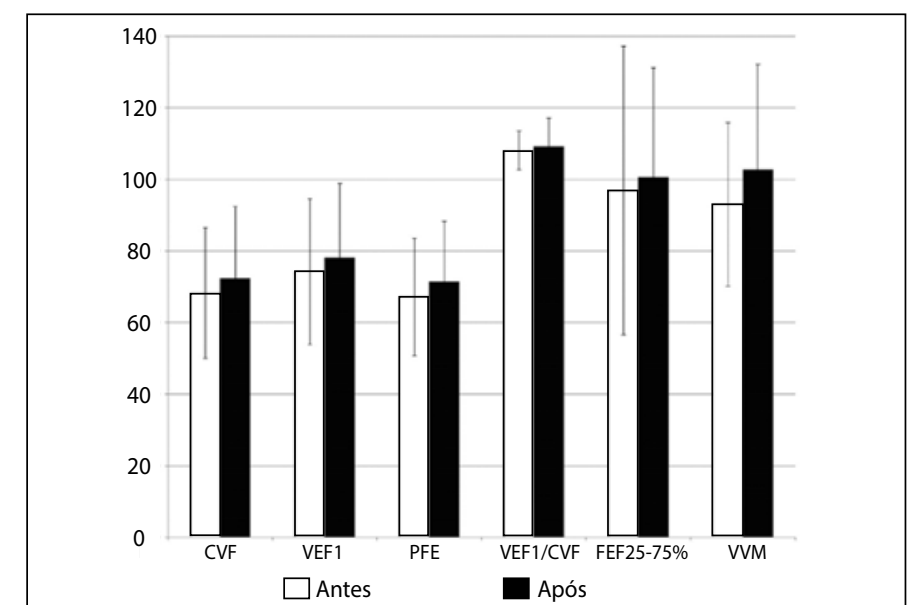

Figura 5. Valores espirométricos em média e desvio padrão antes e após o treinamento, expressos em porcentagem do previsto.

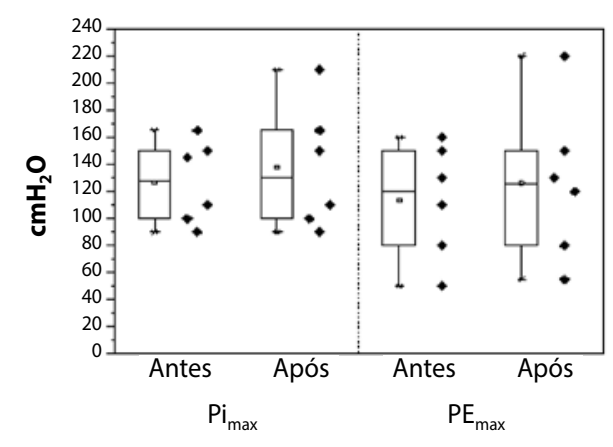

Figura 6. Boxplots para a média, desvio padrão e dispersão da pressão inspiratória máxima (Pimax) e pressão expiratória máxima (Pemax) antes e após o treinamento.

\section{DISCUSSÃO}

Este foi o primeiro estudo que avaliou os efeitos do treinamento com canoagem adaptada sobre a modulação autonômica e função respiratória em paraplégicos. Observou-se após o treinamento aumento da atividade simpática (SD2 e LF em ms²) e parassimpática (rMSSD, pNN50, HF em ms² e SD1), com maior tendência em aumento da atividade parassimpática, porém, sem diferenças estatisticamente significativas. Além disso, a diminuição de LF/HF de 7,0\% e o aumento de SampEn de 20,2\% após o treinamento, refletem uma melhora da modulação simpatovagal. O tamanho reduzido da amostra e a grande variação dos dados podem ter influenciado os resultados.

Os estudos a seguir demonstraram os efeitos do treinamento combinado (aeróbico e força) sobre a VFC de pessoas sem LM. Karavirta et al. ${ }^{23}$ observaram melhora na modulação autonômica cardíaca em homens de meia idade. Camillo et al. ${ }^{24}$ verificaram aumento dos intervalos RR, de SDNN e rMSSD e diminuição da FC em homens idosos com doença pulmonar obstrutiva crônica. Rossi et al. ${ }^{25}$ demonstraram aumento de rMSSD, dos intervalos RR e de HF e diminuição de LF e da FC, demonstrando aumento parassimpático e redução do simpático em mulheres após a menopausa.

Outros estudos demonstraram os efeitos do treinamento aeróbico e de força separadamente sobre o sistema cardiovascular de paraplégicos. Milia et al. ${ }^{26}$ demonstraram aumento da pré e pós-carga, atribuído à melhora no tônus vasomotor que facilita o retorno venoso, isso compensaria a perda simpática, mostrando a importância das adaptações periféricas ao exercício sobre o controle cardiovascular. Nash et al. ${ }^{27}$ demonstraram aumento do $\mathrm{VO}_{2}$ pico em 10,4\%, da potência anaeróbica média e de pico em 8,6\% e 6\%, respectivamente. Nash et al. ${ }^{22}$ compararam os efeitos dois circuitos de treinamento de força (aparelhos e Thera band ${ }^{\circledR}$ ) e observaram que não houve diferença significativa no $\mathrm{VO}_{2}$ e FC entre os treinamentos, porém a percepção de esforço (escala de Borg) foi maior no treinamento com thera band ${ }^{\circledR}$.

Jacobs et al. ${ }^{28}$ avaliaram o efeito agudo de um circuito de treinamento (exercícios resistidos intervalado com exercícios de alta cadência e baixa resistência) em paraplégicos e observaram valores modestos de $\mathrm{FC}$ média e $\mathrm{VO}_{2}$ médio, correspondendo a 49\% e 76,5\% dos valores de pico, respectivamente. A razão de trocas gasosas variou de 0,96 a 1,19, indicando que o protocolo foi intenso e baseia-se principalmente no metabolismo glicolítico. Estes dados revelam que a intensidade do exercício pode estar superestimada em paraplégicos, quando a FC é usada para prescrever a intensidade de trabalho. Este achado é importante, pois atualmente não há orientações sobre a prescrição de exercícios para pessoas com LM.

Taylor e Mc Gruder ${ }^{10}$ avaliaram de forma qualitativa o significado da canoagem no mar para tetraplégicos. Para os voluntários a canoagem representa uma atividade desafiadora, que possibilita interação social de forma igualitária, sensação de competência, uso do tempo de forma útil, e até a redefinição da vida ou de si mesmo. Segundo os autores a canoagem proporciona estímulo motor de movimento, força e resistência, especialmente de membros superiores para remar, estabilização postural, reações de equilíbrio e de rotação do tronco, movimento recíproco bilateral e estímulo sensorial único por ativação do sistema vestibular nos vários planos de movimentos.

Em relação aos dados espirométricos, os resultados demonstram que não houve diferença estatisticamente significativa entre os parâmetros avaliados pré e pós-treinamento. Porém, no presente estudo houve um aumento de 10,4\% para WM após o treinamento. Quando a WM está diminuída, a ventilação necessária para uma determinada intensidade de exercício aumenta, isso reduz a eficiência ventilatória e consequentemente a tolerância ao exercício. Portanto, o ganho na WM representa um aumento da tolerância ao exercício.

Os três primeiros indivíduos apresentaram espirometria normal, enquanto os outros três apresentaram características restritivas, dados semelhantes foram encontrados por Bernard et al. ${ }^{7}$, Schilero et al. ${ }^{4}$, estes autores atribuem as características restritivas à fraqueza neuromuscular respiratória. Foi possível observar que o padrão restritivo apareceu em indivíduos com diferentes níveis de LM (T4, T5 e T9), todas classificadas como lesões completas (AIS A), isso demonstra que a completude pode ter sido mais importante do que o nível da lesão para definir as alterações respiratórias no grupo estudado. Considerando os valores médios, a amostra apresentou valores razoáveis, para VEF1/CVF e FEF 25-75\% próximos da normalidade, porém todos os outros índices estão abaixo do previsto ${ }^{29}$. Rocha et al. $^{30}$ e Mueller et al. ${ }^{31}$ encontraram dados semelhantes em paraplégicos.

Houve um aumento após o treinamento na $\mathrm{Pi}_{\max }$ e $P \mathrm{P}_{\max }$ de 8,5\% e $11,0 \%$, respectivamente, porém não estatisticamente significativo. Foi possível observar grande dispersão dos valores, isso mostra a heterogenia da amostra, mesmo sendo todos paraplégicos, o que provavelmente influenciou os resultados. O valor médio antes do treinamento de $\mathrm{Pi}_{\max }$ de $-126,67 \pm 30,60 \mathrm{CmH}_{2} \mathrm{O}$ está dentro do padrão de normalidade segundo Harik-Khan ${ }^{32}$, porém o valor médio de $\mathrm{Pe}_{\max }$ de 113,33 $\pm 42,26$ $\mathrm{CmH}_{2} \mathrm{O}$ está abaixo da normalidade para idade $(233 \pm 84)^{32}$. Mueller et al. ${ }^{31}$ encontram em indivíduos paraplégicos $\mathrm{Pi}_{\max } 20 \%$ e $\mathrm{Pe}_{\max } 60 \%$ abaixo do previsto. Isso pode ser devido ao comprometimento parcial dos músculos intercostais e abdominais em paraplégicos ${ }^{18}$.

Considerando que os músculos intercostais e abdominais são inervados por raízes nervosas dos segmentos medulares T1-T6 e T5-L1, respectivamente ${ }^{17}$, mesmo os indivíduos com lesão torácica baixa podem apresentar alterações pulmonares, como menor volume ${ }^{7}$ e parâmetros como CVF, VEF1 e PFE abaixo do previsto $^{18}$. 
Para Sheel et al. ${ }^{8}$, os músculos respiratórios apresentam características plásticas tanto estruturalmente como metabolicamente. É possível que os efeitos positivos do treinamento físico possam residir em um aumento da força e resistência muscular, sobretudo da musculatura acessória.

Sugere-se que para se obter melhores resultados sobre a função cardiopulmonar, os indivíduos teriam que ser submetidos a um estímulo de treinamento mais intenso e/ou prolongado. Isso mostra a necessidade de novos estudos, para definir o melhor protocolo de treinamento para esta população, bem como parâmetros de prescrição do treinamento.

Muitos são os desafios encontrados na realização de estudos com pessoas que sofreram LM. Ensaios clínicos randomizados e controlados são considerados padrão ouro em pesquisa científica, porém pode criar grandes desafios metodológicos (dificuldade de compor uma amostra grande e homogênea), éticos (grupo controle de pessoas com LM não envolvido no programa de exercício físico) e práticos (menor assiduidade e abandono, falta acessibilidade, transporte, acompanhante, complicações secundárias como infeções urinárias, úlceras de pressão, dor nos membros superiores) quando aplicado para treinamento físico em pessoas com LM. Por todos estes fatores não é surpreendente que muitos dos estudos realizados com estes indivíduos apresentam baixo poder estatístico para detectar diferenças estatisticamente significantes. Por isso, estudos que demonstram até mesmo pequenos efeitos em amostras altamente heterogêneas são muito valiosos para demonstrar o impacto potencial do exercício físico ${ }^{33}$.

\section{CONCLUSÃO}

O protocolo proposto de três meses de canoagem adaptada não foi capaz de promover efeitos significativos sobre os parâmetros cardiopulmonares avaliados em indivíduos paraplégicos pós-LM, porém se observou uma tendência em melhora da maioria destes parâmetros, principalmente os referentes à VFC, neste sentido, provavelmente o estímulo de treinamento tenha sido insuficiente.

$\overline{\text { Todos os autores declararam não haver qualquer potencial conflito }}$ de interesses referente a este artigo.

CONTRIBUICÕES DOS AUTORES: Cada autor contribuiu individual e significativamente para o desenvolvimento do manuscrito. EMCA (0000-0002-4370-8610)*, RSA (0000-0003-47983852)* e LOP (0000-0001-52736261)* foram os principais contribuintes nas avaliações, coletas de dados, aplicação do protocolo de treinamento e redação do manuscrito. EMCA e ARPJ (0000-0002-4217-7328)* fizeram as análises estatísticas. AMCA, ARPJ, FPSL (0000-0001-9834-7800)* e MOL (0000-0001-9990-5296)* realizaram a revisão e aprovação final do manuscrito. *ORCID (Open Researcher and Contributor ID).

\section{REFERÊNCIAS}

1. Oh YM, Eun JP. Cardiovascular dysfunction due to sympathetic hypoactivity after complete cervical spinal cord injury: a case report and literature review. Medicine (Baltimore). 2015;94(12):e686.

2. Malmqvist L, Biering- Sørensen T, Bartholdy K, Krassioukov A, Welling K-L, Svendsen JH et al. Assessment of autonomic function after acute spinal cord injury using heart rate variability analyses. Spinal Cord. 2015;53(1):54-8.

3. Claydon VE, Krassioukov AV. Clinical correlates of frequency analyses of cardiovascular control after spinal cord injury. Am J Physiol Heart Circ Physiol. 2008;294(2):H668-78.

4. Schilero GJ, Spungen AM, Bauman WA, Radulovic M, Lesser M. Pulmonary function and spinal cord injury. Respir Physiol Neurobiol. 2009;166(3):129-141.

5. Merati G, Di Rienzo M, Parati G, Veicsteinas A, Castiglioni P. Assessment of the autonomic control of heart rate variability in healthy and spinal-cord injured subjects: contribution of different complexity-based estimators. IEEE Trans Biomed Eng. 2006;53(1):43-52.

6. Agiovlasitis S, Heffernan KS, Jae SY, Ranadive SM, Lee M, Mojtahedi MC, et al. Effects of paraplegia on cardiac autonomic regulation during static exercise. Am J Phys Med Rehabil. 2010;89(10):817-23.

7. Bernard PL, Mercier J, Varray A, Prefaut C. Influence of lesion level on the cardioventilatory adaptations in paraplegic wheelchair athletes during muscular exercise. Spinal Cord. 2000;38(1):16-25.

8. Sheel AW, Reid WD, Townson AF, Ayas NT, Konnyu KJ. Effects of exercise training and inspiratory muscle training in spinal cord injury: A systematic review. J Spinal Cord Med. 2008;31(5):500-8.

9. Postma K, Haisma JA, de Groot S, Hopman MT, Bergen MP, Stam HJ et al. Changes in pulmonary function during the early years after inpatient rehabilitation in persons with spinal cord injury: a prospective cohort study. Arch Phys Med Rehabil. 2013;94(8):1540-6.

10. Taylor LPS, McGruder JE. The meaning of sea kayaking for persons with spinal cord injuries. Am J Occup Ther. 1996;50(1):39-46.

11. Carneiro LM, Castro FAS. Cinemática da canoagem: revisão. R Bras Ci e Mov. 2009;17(3):114-22.

12. Nakamura FY, Cyrino ES, Borges TO, Okano AH, Melo JC, Fontes EB. Variação dos parâmetros do modelo de potência crítica em resposta a treinamento de canoagem. Rev. Bras. Cineantropom. Desempenho Hum. 2006;8(2):5-12

13. Nakamura FY, Borges TO, Sales OR, Cyrino ES, Kokubun E. Estimativa do custo energético e contribuição das diferentes vias metabólicas na canoagem de velocidade. Rev Bras Med Esporte. 2004;10(2):70-77.

14. Millar PJ, Rakobowchuk M, Adams MM, Hicks AL, McCartney N, MacDonald MJ. Effects of short-term training on heart rate dynamics in individuals with spinal cord injury. Auton Neurosci. 2009;150(1-2):116-21.

15. Brennan M, Palaniswami M, Kamen P. Do existing measures of Poincare plot geometry reflect nonlinear features of heart rate variability? IEEE Trans Biomed Eng. 2001;48(11):1342-7.

16. Pereira CA. Espirometria. J Pneumol 2002;28 (Supl 3):S1-S82.

17. Paleville DGT, McKay WB, Folz RJ, Ovechkin AV. Respiratory motor control disrupted by spinal cord injury: mechanisms, evaluation, and restoration. Transl Stroke Res. 2011;2(4):463-73.
18. Mueller G, de Groot S, van der Woude L, Hopman MT. Time-courses of lung function and respiratory muscle pressure generating capacity after spinal cord injury: a prospective cohort study. J Rehabil Med. 2008;40(4):269-76.

19. Gastaldi AC, Freitas Filho GA, Pereira APM, Silveira JM. Quantas medidas de pressões respiratórias são necessárias para se obterem medidas máximas em pacientes com tetraplegia? Coluna/Columna. 2010;9(2):138-44

20. Neder JA, Andreoni S, Lerario MC, Nery LE. Reference values for lung function tests. II. Maximal respiratory pressures and voluntary ventilation. Braz J Med Biol Res. 1999;32(6):719-27.

21. Tawashy AE, Eng JJ, Krassioukov AV, Miller WC, Sproule S. Aerobic exercise during early rehabilitation for cervical spinal cord injury. Phys Ther. 2010;90(3):427-37.

22. Nash MS, Jacobs PL, Woods JM, Clark JE, BS, Pray TA, Pumarejo AE. A Comparison of 2 circuit exercise training techniques for eliciting matched metabolic responses in persons with paraplegia. Arch Phys Med Rehabil. 2002;83(2):201-9.

23. Karavirta L, Tulppo MP, Laaksonen DE, Nyman K, Laukkanen RT, Kinnunen H, et al. Heart rate dynamics after combined endurance and strength training in older men. Med Sci Sports Exerc. 2009;41 (7):1436-43

24. Camillo CA, Laburu VM, Gonçalves NS, Cavalheri V, Tomasi FP, Hernandes NA, et al. Improvement of heart rate variability after exercise training and its predictors in COPD. Respir Med. 2011;105(7):1054-62.

25. Rossi FE, Ricci-Vitor AL, Buonani CS, Vanderlei LCM, Freitas Junior IF. The Effects of combined aerobic and resistance training on heart rate variability in postmenopausal women. Medicina (Ribeirão Preto). 2013;46(2):171-7.

26. Milia R, Roberto S, Marongiu E, Olla S, Sanna I, Angius L, et al. Improvement in hemodynamic responses to metaboreflex activation after one year of training in spinal cord injured humans. Biomed Res Int. 2014;893468:9p.

27. Nash MS, van de Ven I, van Elk N, Johnson BM. Effects of circuit resistance training on fitness attributes and upper-extremity pain in middle-aged men with paraplegia. Arch Phys Med Rehabil. 2007;88:70-5.

28. Jacobs PL, Mahoney ET, Nash MS, Green BA. Circuit resistance training in persons with complete paraplegia. J Rehabil Res Dev. 2002;39(1):21-8.

29. Neder JA, Andreoni S, Castelo-Filho A, Nery LE. Reference values for lung function tests. I. Static volumes. Braz J Med Biol Res. 1999;32(6):703-17.

30. Rocha AP, Mateus SRM, Horan TA, Beraldo PSS. Determinação não-invasiva da pressão inspiratória em pacientes com lesão medular traumática: qual é o melhor método? J Bras Pneumol. 2009;35(3):256-60.

31. Mueller $G$, Perret C, Spengler CM. Optimal intensity for respiratory muscle endurance training in patients with spinal cord injury. J Rehabil Med. 2006;38(6):381-86.

32. Harik-Khan RI, Wise RA, Fozard J. Determinants of maximal inspiratory pressure. The Baltimore Longitudinal Study of Aging. Am J Respir Crit Care Med. 1998;158(5 pt 1):1459-64.

33. Ginis KA, Hicks AL. Exercise research issues in the spinal cord injured population. Exerc Sport Sci Rev 2005;33(1):49-53 
Anexo 1. Protocolo de treinamento.

\begin{tabular}{|c|c|c|}
\hline Primeiro mês & Segundo mês & Terceiro mês \\
\hline $\begin{array}{l}\text { a) Remadas Vai frente e volta ré remando com as mãos, } \\
\text { por } 1^{\prime} ;\end{array}$ & a) Remadas vai frente e volta em ré, por 2'; & a) Remadas vai frente e volta em ré $3^{\prime}$; \\
\hline $\begin{array}{l}\text { b) Exercício de rolar a bola a frente no caiaque, por } \\
8 \text { repetições; }\end{array}$ & $\begin{array}{l}\text { b) Exercício isotônico guiado com bola, } 6 \text { repetições } \\
\text { em } 3 \text { direções (frente/direita/esquerda); }\end{array}$ & $\begin{array}{l}\text { b) Exercício de torção do tronco utilizando um sandbag, } \\
6 \text { repetições para em cada direção (horário/anti- } \\
\text { horário); }\end{array}$ \\
\hline c) Ramadas em avanço lateral, por 1> cada lado; & c) Remadas em avanço lateral por 2'; & c) Remadas em avanço lateral, por 3'; \\
\hline $\begin{array}{l}\text { d) Exercício de descarga de peso em MMSS no caiaque } \\
\text { elevando o quadril, por } 5 » \text { em } 3 \text { repetições; }\end{array}$ & $\begin{array}{l}\text { d) Exercício de descarga de peso em MMSS no caiaque } \\
\text { elevando o quadril, por } 5^{\prime \prime} \text { em } 3 \text { repetições, sustentar } \\
\text { e pendular; }\end{array}$ & $\begin{array}{l}\text { d) Exercício de descarga de peso em MMSS no caiaque } \\
\text { elevando o quadril, por } 5 \text { » em } 3 \text { repetições, sustentar } \\
\text { e pendular; }\end{array}$ \\
\hline $\begin{array}{l}\text { e) Remadas a frente unilateral (uma única pá), } \\
\text { alternando os lados, por } 1 \text {; }\end{array}$ & $\begin{array}{l}\text { e) Remadas a frente unilateral (uma única pá), } \\
\text { alternando os lados, por } 2^{\prime} ;\end{array}$ & $\begin{array}{l}\text { e) Remadas a frente unilateral (uma pá só), alternando } \\
\text { os lados, por 3'; }\end{array}$ \\
\hline $\begin{array}{l}\text { f) Isometria utilizando o remo em cada direção (direita/ } \\
\text { esquerda/cima/frente), por 20» a 30»; }\end{array}$ & $\begin{array}{l}\text { f) Exercícios isométrico com Thera band em } 4 \text { direções } \\
\text { (diagonais, frente e cima), } 15 \text { repetições cada; }\end{array}$ & $\begin{array}{l}\text { f) Exercícios isométricos com Thera band em } 4 \text { direções } \\
\text { (diagonais, frente e cima), } 15 \text { repetições cada; }\end{array}$ \\
\hline $\begin{array}{l}\text { g) Remadas a frente com fase aérea rápida e pouca } \\
\text { tração na água, por } 1{ }^{\prime} ;\end{array}$ & g) Remadas a frente com o máximo de ADM, por 2> & $\begin{array}{l}\text { g) Tapa cruzado com o remo em uma prancha de EVA } \\
\text { a frente, em } 3 \text { séries de } 4 \text { repetições para cada direção, } \\
\text { frente baixa e frente alta; }\end{array}$ \\
\hline h) Arremessos de bola, por 2خ; & h) Arremessos de bola, por 3;; & h) Remadas em ré com torção do tronco, por 3'; \\
\hline $\begin{array}{l}\text { i) Balanço com um tapa de remo rápido para o lado } \\
\text { que balançar (Low Brace), por 1'cada braço; }\end{array}$ & $\begin{array}{l}\text { i) Remadas a frente utilizando impulso com o tronco, } \\
\text { por } 2 ; \text {; }\end{array}$ & $\begin{array}{l}\text { i) Remadas a frente alternando rápido por } 20 » \text { e lento } \\
\text { por } 20 » \text {, durante } 3 ; \text {; }\end{array}$ \\
\hline $\begin{array}{l}\text { j) Remadas a frente alternando com avanço lateral e ré } \\
\text { até dar o tempo do treino. }\end{array}$ & $\begin{array}{l}\text { j) Remadas a frente alternando rápido por } 20^{\prime \prime} \text { e lento } \\
\text { por } 20^{\prime \prime} \text {, durante } 2^{\prime} \text {. }\end{array}$ & $\begin{array}{l}\text { j) Remadas a frente utilizando impulso com o tronco, } \\
\text { alternando com avanço lateral e ré até dar o tempo } \\
\text { do treino. }\end{array}$ \\
\hline
\end{tabular}

Entre cada exercício houve um intervalo de cerda de 1 minuto.

minuto; "segundo; MMSS: membros superiores. 